\title{
Current treatment options for recurrent nasopharyngeal cancer
}

\author{
Carlos Suárez • Juan P. Rodrigo • Alessandra Rinaldo • \\ Johannes A. Langendijk $\cdot$ Ashok R. Shaha $\cdot$ Alfio Ferlito
}

Received: 19 July 2010 / Accepted: 27 August 2010 / Published online: 24 September 2010

(C) The Author(s) 2010. This article is published with open access at Springerlink.com

\begin{abstract}
Loco-regional control rate of nasopharyngeal carcinoma (NPC) has improved significantly in the past decade. However, local recurrence still represents a major cause of mortality and morbidity in advanced stages, and management of local failure remains a challenging issue in NPC. The best salvage treatment for local recurrent NPC remains to be determined. The options include brachytherapy, external radiotherapy, stereotactic radiosurgery, and nasopharyngectomy, either alone or in different combinations. In this article we will discuss the different options for salvage of locally recurrent NPC. Retreatment of locally recurrent NPC using radiotherapy, alone or in combination
\end{abstract}

This paper was written by members of the International Head and Neck Scientific Group (www.IHNSG.com)

\section{Suárez · J. P. Rodrigo}

Instituto Universitario de Oncología del Principado de Asturias,

Oviedo, Spain

C. Suárez · J. P. Rodrigo

Department of Otolaryngology,

Hospital Universitario Central de Asturias, Oviedo, Spain

A. Rinaldo · A. Ferlito $(\square)$

Department of Surgical Sciences, ENT Clinic,

Azienda Ospedaliero-Universitaria,

Piazzale S. Maria della Misericordia, 33100 Udine, Italy

e-mail: a.ferlito@uniud.it

\section{J. A. Langendijk}

Department of Radiation Oncology,

University Medical Center Gröningen,

University of Gröningen, Groningen, The Netherlands

\section{A. R. Shaha}

Head and Neck Service,

Memorial Sloan-Kettering Cancer Center,

New York, NY, USA with other treatment modalities, as well as surgery, can result in long-term local control and survival in a substantial proportion of patients. For small-volume recurrent tumors (T1-T2) treated with external radiotherapy, brachytherapy or stereotactic radiosurgery, comparable results to those obtained with surgery have been reported. In contrast, treatment results of advanced-stage locally recurrent NPC are generally more satisfactory with surgery (with or without postoperative radiotherapy) than with reirradiation.

Keywords Nasopharyngeal carcinoma $\cdot$ Recurrent . Radiotherapy $\cdot$ Brachytherapy $\cdot$ Nasopharyngectomy

\section{Introduction}

Loco-regional control rate of nasopharyngeal carcinoma (NPC) has improved significantly in the past decade due to the advances in imaging, radiotherapy techniques, and the use of combined treatments with chemoradiotherapy. At present, the reported 5-year local control rates of NPC ranges from 80 to $85 \%$. Despite the improved outcome in local control, local recurrence still represents a major cause of mortality and morbidity in advanced stages, and management of local failure remains a challenging issue in NPC.

\section{Diagnosis of recurrence}

Early detection of persistent or recurrent disease is of paramount importance. The most frequently used methods include image techniques [computed tomography (CT), magnetic resonance imaging (MRI)], rigid nasopharyngeal endoscopy and nasopharyngeal biopsies, and serological tests for immunoglobulin A against Epstein-Barr virus 
(EBV). For patients with elevated anti-EBV titers or suspicious endoscopic examination, a biopsy of the nasopharynx is then indicated to detect the recurrence in its early stages. Rigid endoscopic examination provides a better view than flexible endoscopy, to the extent that even submucosal bulges can be seen. CT and MRI are performed on patients with a confirmed diagnosis. CT scan delineates the extent of tumor in the nasopharynx and whether it has affected surrounding bony structures. MRI, aside from providing better images of soft tissue, also shows tumor size and extension to the skull base and intracranial cavity. Initial assessment is better carried out by rigid nasopharyngeal endoscopy; according to prospective trials comparing it to CT shows a significantly better agreement with the histological findings $[1,2]$.

A paraneoplastic syndrome (PNS) often represents the only signal of a silent neoplasm and sometimes it precedes the tumor itself. PNS may precede the clinical manifestation of a persistent or recurrent NPC or of asymptomatic metastases. PNS subsides in parallel to response of the NPC, and thus may be useful for the diagnosis of recurrence. These syndromes can follow the clinical course of the tumor and thus can be useful for monitoring its evolution [3].

In addition to the most frequent local recurrences, treatment failures can be observed in the neck. The detection of the cervical lymph node metastases on presentation has improved with MRI and positron emission tomography (PET), and can be cytologically confirmed through fine needle aspiration cytology.

In a series of 891 patients with recurrent NPC, only $70 \%$ of patients had local failure alone, as $25 \%$ also suffered from regional relapse and $8 \%$ from distant metastasis [4].

If the tumor recurs or persists in the nasopharynx after radiotherapy, then salvage procedures are still an option if there is no distant metastasis. In the next sections we will discuss the different options for salvage of recurrent NPC. Due to the significantly better local control and survival rates for patients with persistent disease [5], only local recurrent tumors will be considered.

\section{External beam radiotherapy}

\section{Results of retreatment and influencing factors}

Despite radical irradiation, local recurrence remains a major problem for patients with NPC. The reported incidence varies from 8 to $58 \%$, with the median at $34 \%$ [4].

Locally persistent or recurrent NPC may be due to the intrinsic radio-resistance of the primary tumor, insufficient dose, or a geographic miss during the initial radiotherapy. The results of initial treatment with radiation therapy have improved over the years due to the advances in diagnostic imaging, which help to localize the full extent of the primary tumor, the higher tumor doses delivered [6], the different regimes of fractionation, the use of advanced radiation delivery techniques, such as intensity-modulated radiation therapy (IMRT) [7-9], and the use of concomitant chemotherapy [10].

Pretreatment evaluation of the extent of disease, including paranasopharyngeal extension, is crucial. Early detection is important as the chance of salvage varies with the extensiveness of local recurrence. In the series of Lee et al. [4] the 5-year local control rate for rT3 patients was only $11 \%$ compared to 35 and $28 \%$ for rT1 and rT2, respectively, which also translated into worse overall survival for rT3 patients (4\%) as compared to for rT1 (27\%). In Wang's series [11], the 5-year survival was $38 \%$ for patients with rT1-T2 tumors and $15 \%$ for those patients with rT3-T4. Overall 5-year actuarial local control, and overall survival rank from 15 to $35 \%$, and 8 to $41 \%$, respectively [4, 11-14].

These results emphasize the importance of close followup after primary treatment and more vigorous attempts for early detection of local recurrence by rigid nasopharyngeal endoscopy [15], CT and/or MRI. Recent studies indicate that PET using 18-fluoro-2-deoxyglucose (FDG), is more accurate in detecting local recurrence after curative (chemo) radiation than MRI $[16,17]$ and/or may add useful additional information to disputable findings on MRI [18, 19]. Nevertheless, we must consider the difficulty in imaging near the brain, which is always hypermetabolic, and the difficulties with local inflammation secondary to otitis media and bony necrosis of the skull base related to radiation.

In some reports there is a polarization of primary radiotherapy and reirradiation into two extremes (good results in primary treatment and poor results in reirradiation, and viceversa) which is most likely due to the successful prevention of avoidable local failures during primary radiotherapy and the natural selection of the most radioresistant tumors for reirradiation [14].

It is of interest to remark that although patients with undifferentiated carcinomas have a notably higher 5-year survival than those with squamous cell carcinomas, in some series there is no difference when considering 10-year survival [12]. This longer survival may be due to slower regrowth of undifferentiated carcinomas, evident in their longer time to local recurrence, as well as that patients with undifferentiated carcinomas are more likely to develop late distant metastases. The stage at which recurrence is diagnosed is the most significant prognostic factor, but lesions involving the skull base should be differentiated from other advanced-stage tumors when evaluating tumor control or survival [20, 21].

Furthermore, patients without evidence of intracranial invasion or cranial nerve palsy have better survival than those with such lesions (3-year survival, 31 vs. 4\%) [20]. 
Other factors adversely affecting survival are the simultaneous presence of neck metastases and patients whose tumor recurred at 1-2 years or less after initial treatment [11, 22].

Irrespective of the total dose given during initial treatment, there is significant correlation between the dose at reirradiation and the salvage rate. The highest local control rates have been achieved with a total dose of at least $60 \mathrm{~Gy}$ $[4,11,14,20]$.

Nevertheless, data from retrospective studies must be interpreted cautiously when comparing treatment techniques because unrecognized factors may have been used to select the type of treatment.

\section{Complications of retreatment}

Different kinds of complications have been reported in patients undergoing reirradiation, ranging from 6 to $85 \%$ $[4,11,13,14,20,22]$. This enormous range can be explained by the different reirradiation doses and techniques used as well as by the fact that some authors only report severe complications. The most frequent complications include xerostomia, trismus, sensorineural hearing loss, and middle ear effusion, but also brain necrosis which has been reported up to $20 \%$ of patients $[4,11,13,14,20$, 22]. Fatal complication rates between 2 and $10 \%$ have also been reported [4, 12, 14, 23]. The incidence of severe complications depends on the dose received and treatment modality. Patients who were retreated with a cumulative external beam dose $>100$ Gy had a high incidence of severe complications: $4 \%$ for patients receiving doses less than or equal to $100 \mathrm{~Gy}$ compared with $39 \%$ for those patients who received doses greater than $100 \mathrm{~Gy}$ [12]. Lee et al. [24] reported on the largest series of 654 local recurrent NPC patients treated with reirradiation. In their series, the risk of late complications was predominantly affected by the biological effective dose of the primary treatment, while the corresponding impact of reirradiation failed to reach statistical significance, indicating higher tolerance for reirradiation when primary treatment has been carried out with better sparing of normal tissues.

External reirradiation of NPC with curative intent is often difficult due to the large number of nearby critical structures that were previously irradiated to a high dose. Conventional external radiotherapy delivered by a 10-MV photon beam with bilateral opposing fields to cover the recurrent tumor site and margins yields a higher complications rate than three-dimensional (3D) conformal radiotherapy or IMRT, which try to radiate the previously radiated areas (such as temporal lobes, temporo-mandibular joints and middle ears) at less than $60 \%$ of the prescribed dose. Thus, Chang et al. [20] observed severe complications in $23 \%$ of the conventional radiotherapy group, but only in $9 \%$ of the conformal radiotherapy group. Furthermore, with conventional external radiotherapy, 13 patients $(14 \%)$ were found to have brain necrosis, and no brain necrosis occurred in patients treated with conformal radiotherapy, although the lower incidence of complications they saw in patients treated with conformal radiotherapy may also be explained by a shorter follow-up [20].

For patients with extensive disease requiring external radiotherapy, superior dose distribution and organ sparing have been demonstrated with IMRT over more conventional conformal radiation delivery techniques for salvage of NPC. Excellent local control rates have been reported using IMRT for newly diagnosed NPC [25, 26], but although Lu et al. [27] have reported good local control rate after high-dose IMRT (68-70 Gy) for recurrent NPC, the relatively short follow-up makes it difficult to evaluate late toxicities in their patients. Late toxicities are still common after IMRT, although generally most are mild. Neurological toxicity represents the main complication but it appears to be less frequent than after conventional two-dimensional (2D) or 3D conformal reirradiation [28]. Longer follow-up of patients is still needed to address more clearly the incidence and impact of late toxicities.

\section{Brachytherapy}

What is the best salvage treatment for early-stage recurrent NPC? It is an unanswered question. The options include brachytherapy alone with either interstitial or intracavitary approach, external radiotherapy with/without brachytherapy, stereotactic radiosurgery, and nasopharyngectomy. The last two options will be developed in other sections.

The inherent physical and dosimetric characteristics of brachytherapy allow the delivery of a high dose to the nasopharynx while minimizing the dose to the adjacent structures.

For small-volume recurrences treated with brachytherapy alone, Choy et al. [29] reported a local control rate of $61 \%$ at 5 years with 60 Gy delivered by gold grain implants. Moreover, the local control was superior in failures confined to the nasopharynx compared to failures extending beyond the nasopharynx, 81 versus $44 \%$, respectively. Similar results have been observed by Kwong et al. [5] who observed an overall survival rate at 5 years of $54 \%$, with the sequelae of headache, palatal fistula, and mucosal radiation necrosis at the site of implantation in 28,19 , and $16 \%$ of the patients, respectively.

Patients with large volume local recurrence will benefit from external radiotherapy with or without brachytherapy. Taking into account that a small increase in external radiotherapy dose may result in marked increase in toxicity, for rT1-T2 local recurrences combined treatment is recommended as shown by its superior local control and relatively lower risk of complications. With the advances in 
imaging technology and the advent of 3D conformal techniques, it is possible to reduce the target volume without jeopardizing the local control, and the complication rates should be reduced. After the completion of external radiotherapy, a routine brachytherapy boost can improve the local control of patients with T1-T2 disease. However, treatment results of advanced-stage locally recurrent NPC has been unsatisfactory either with external radiotherapy or brachytherapy, alone or combined [24].

Thus, Leung et al. [23] compared the results of external radiotherapy alone and combined with brachytherapy. Subgroup analysis on the external radiotherapy and combined treatment groups showed a 3-year local failure-free survival rate of 33 and $57 \%$, respectively, but external radiotherapy group had an excess of patients with rT3 disease. Further analysis was performed on the rT1-T2 patients showing a trend toward improvement in local control in favor of the combined treatment group (3-year local failure-free survival rate of $72 \%$ with combined treatment; $54 \%$ with external radiotherapy). Multivariate analyses showed that $\mathrm{rT}$ stage and total equivalent dose were significant predicting factors of success. Concerning major and central nervous system complications, modality of treatment (more complications with external radiotherapy group) and rT stage were significant for predicting the occurrence of such complications [23].

On the other hand, Zheng et al. [30] reported that the 5year actuarial local failure-free survival rate of patients with initially diagnosed T3-T4 disease for the 3D conformal radiotherapy group and brachytherapy group was 84 versus $60 \%$, with $3 \mathrm{D}$ conformal radiotherapy providing better local control than brachytherapy as a salvage treatment for locally persistent NPC, especially in patients with initially diagnosed T3-T4 disease.

These results highlight the importance of early detection of local recurrences. The primary influence is a factor independently significant after adjustment for rT-stage effect. In the Leung et al. [23] series, $65 \%$ of rT3 recurrence originated from a $\mathrm{T} 3$ primary.

The possibility of adopting other approaches, such as concurrent chemoradiation or accelerated fractionation, which have been shown to improve the treatment outcome in the primary treatment of T3-T4 disease, should be seriously considered.

\section{Stereotactic radiosurgery}

Fundamentals and results of treatment

Because of the poor results of conventional external reirradiation, other techniques of reirradiation have been employed for locally recurrent NPC, such as stereotactic radiosurgery. Stereotactic radiosurgery is a highly precise form of radiation therapy used primarily to treat tumors and other abnormalities of the brain. Despite its name, stereotactic radiosurgery is a non-surgical procedure that delivers a single high dose of precisely targeted radiation using highly focused gamma-ray or X-ray beams that converge on the specific area or areas of the brain where the tumor or other abnormality resides, minimizing the amount of radiation to healthy brain tissue. Although stereotactic radiosurgery is often completed in a 1-day session, sometimes fractionated stereotactic treatment is applied, especially for larger tumors. This procedure is usually referred to as fractionated stereotactic radiotherapy in case more than five fractions are given.

Several series have reported satisfactory local control when stereotactic radiosurgery was used to treat recurrent NPC, as shown by Chua et al. [31] who reported a 5-year overall survival rate of $47 \%$. The time interval from primary radiotherapy, rT stage, prior local failures and tumor volume were significant predictive factors for local control and/or survival. A radiosurgery prognostic scoring system was designed based on these predictive factors. Five-year local failure-free probabilities in patients with good, intermediate and poor prognostic scores were 100, 42.5, and $9.6 \%$, respectively. The corresponding 5-year overall survival rates were $100,51.1$, and $0 \%$ [31].

It has been postulated that, combining stereotactic radiosurgery with high-dose-rate brachytherapy might improve the survival because the single large dose of radiosurgery may increase tumor cell kill overcoming the inherent radioresistance of cells. Low et al. [32] treated 36 patients with local recurrent NPC in stage rT1-T2 with a schedule of 18 Gy followed by two separate fractions of 6 Gy each by intracavitary brachytherapy. The actuarial 5-year disease-free survival and overall survival were $57 \%$ (78\% for rT1, and $39 \%$ for rT2) and $62 \%$ ( $80 \%$ for rT1, and $48 \%$ for rT2), respectively. However, $44 \%$ of patients had late complications, including cranial nerve palsies (20\%), temporal lobe necrosis $(8 \%)$, and osteoradionecrosis of the skull base (17\%).

A matched cohort study to select patients with similar characteristics treated by stereotactic radiosurgery (median dose $12.5 \mathrm{~Gy}$ ) or intracavitary irradiation (total dose of $60 \mathrm{~Gy}$ ) was carried out by Chua et al. [33] in 74 patients with local NPC failure, generally at stage rT1-T2. The 3year local failure-free rate was $78 \%$ for the radioactive gold grain group compared with $68 \%$ for the stereotactic radiosurgery group, whereas the overall survival rate was better in the stereotactic radiosurgery group (3-year survival rate of 77 vs. 66\%), but in both circumstances the differences were not statistically significant. Furthermore, when the impact of tumor volume on treatment outcome was adjusted, no difference in tumor control was observed between the two groups, suggesting that both salvage treatments have comparable efficacy. The incidence of compli- 
cations was similar in number in both groups, but complications in the stereotactic radiosurgery group were more severe $(21.6 \%$ of neuroendocrine complications, $13 \%$ of brain necrosis, $5 \%$ of cranial neuropathy, $5 \%$ of pituitary insufficiency, and severe hemorrhage from a carotid artery aneurysm in one patient versus $30 \%$ of headaches, $16 \%$ of palatal fistula, and $13 \%$ of neuroendocrine complications in the brachytherapy group) [33]. We can ask ourselves if we are making any progress with the new and more sophisticated procedures.

Stereotactic radiosurgery has also been used as a boost after reirradiation with external beam for recurrent NPC with an overall 5-year survival rate of $31 \%$ [34].

\section{Complications of treatment}

Severe complications commonly reported after stereotactic radiosurgery for NPC include massive epistaxis, nasopharyngeal necrosis, cranial nerve palsies, temporal lobe necrosis, and osteoradionecrosis of skull base. The relatively high risk of severe late complications indicates that careful patient selection and treatment planning are required. Severe complications were also reported by Kocher et al. [35], who treated 8 patients with recurrent NPC by stereotactic radiosurgery. In Kocher's series, 3 patients died of carotid or cerebral hemorrhage after stereotactic radiosurgery using a dose of 15-24 Gy, 2 patients developed cerebral edema in temporal lobes, and 1 developed cranial neuropathy. A fatal carotid artery hemorrhage also was the cause of dead of $33 \%$ of patients with recurrent NPC treated with fractionated stereotactic radiotherapy [36]. Tumors involving the Rosenmueller's fossa and invading deeply to the foramen lacerum are the most important predisposing factor in fatal hemorrhage [36]. It should be noted that the large doses per fraction used in most series on stereotactic radiosurgery may cause these relatively high rates of late complications [24].

As previously mentioned, fractionated stereotactic radiotherapy is a modification of stereotactic radiosurgery, which enables fractionated irradiation to be given without losing the advantage of the mechanical precision and accuracy as well as dose conformity of stereotactic radiosurgery. Compared with stereotactic radiosurgery using single fraction of highdose irradiation, fractionated stereotactic radiotherapy may be superior in terms of tumor control and protection of normal tissues and organs surrounding the target.

Based on these principles, 56 patients with recurrent NPC (rT1-T2, 38; rT3, 18) received fractionated stereotactic radiotherapy. The median fractionated stereotactic radiotherapy dose was $48 \mathrm{~Gy}$ in six fractions. Three-year local failure-free survival, disease-specific survival, and progression-free survival rates were 75.1, 45.9, and $42.9 \%$, respectively. Multivariate analysis showed that recurrent disease and large tumor volume were independent factors that predicted poorer disease-specific survival. Seventeen patients developed late complications, including two with fatal hemorrhage [37]. Severe late complications occurred in $25 \%$ of patients with recurrent NPC. Four percent of patients with recurrent disease developed massive hemorrhage in the nasopharynx after fractionated stereotactic radiotherapy and died of this complication, and $6 \%$ developed brain stem necrosis [37].

In a study on 125 NPC patients who received salvage stereotactic radiation Chua et al. [38] compared the results of stereotactic radiation using single or multiple fractions. All patients were individually matched for $\mathrm{rT}$ stage, and tumor volume. The median dose was $12.5 \mathrm{~Gy}$ in single fraction by stereotactic radiosurgery, and $34 \mathrm{~Gy}$ in $2-6$ fractions by fractionated stereotactic radiotherapy. Local control rate was better in the last group although overall survival rates were similar (3-year overall survival rates of 66 and 61\%, respectively). Finally, incidence of severe late complications was $33 \%$ in the stereotactic radiosurgery group versus $21 \%$ in the fractionated stereotactic radiotherapy group, including brain necrosis (16 vs. 12\%) and hemorrhage (5 vs. $2 \%$ ) [38].

\section{Radiotherapy with protons}

Irradiation with protons instead of the currently used photons generally results in a significantly lower physical dose in the co-irradiated healthy tissues, due to its superior beam properties. In practice, proton beams are typically manipulated to generate a spreadout Bragg peak to yield a flat beam depth profile across the target followed by a rapid fall to zero dose, thereby producing little or no exit irradiation. The Bragg peak associated with charged particle beams is extremely useful when attempting in treating a tumor which directly overlies vulnerable normal tissue. Although no clinical data on reirradiation in NPC are available yet, recent studies comparing dose distribution that can be achieved with protons compared to photons in NPC indicate superior dose coverage of the target volume with significant better sparing of critical organs [39]. In this regard, reirradiation using protons may be a very attractive radiation delivery technique for local recurrent NPC.

Table 1 [4, 12, 14, 20, 22, 23, 32, 34] highlights the results of reirradiation in patients with recurrent NPC.

\section{Role of chemotherapy}

Different randomized trials fulfilling strict entry criteria have compared different regimes of chemoradiotherapy 
Table 1 Results with reirradiation

\begin{tabular}{|c|c|c|c|c|c|c|}
\hline Author & $\begin{array}{l}\text { No. of } \\
\text { cases }\end{array}$ & $\begin{array}{l}\text { Local } \\
\text { control }(\%)\end{array}$ & $\begin{array}{l}5 \text {-year } \\
\text { survival }(\%)\end{array}$ & $\begin{array}{l}\text { Total } \\
\text { complications }(\%)\end{array}$ & $\begin{array}{l}\text { CNS } \\
\text { complications (\%) }\end{array}$ & $\begin{array}{l}\text { Mortality } \\
(\%)\end{array}$ \\
\hline Lee et al. [4] (ERT) & 706 & 32 & 17 & 24 & 6 & 2 \\
\hline Chang et al. [20] (ERT) & 186 & & 12 & 23 & 14 & 0 \\
\hline Hwang et al. [22] (ERT/B/SRS) & 64 & 40 & 37 & 45 & 6 & 0 \\
\hline Pryzant et al. [12] (ERT) & 53 & 35 & 21 & 16 & 6 & 10 \\
\hline Teo et al. [14] (ERT) & 103 & 15 & 8 & 85 & 20 & 3 \\
\hline Leung et al. [23] (ERT/B) & 82 & 38 & 30 & 57 & 29 & 5 \\
\hline Low et al. [32] (SRS) & 36 & 65 & 62 & 44 & 8 & 0 \\
\hline Pai et al. [34] (ERT/SRS) & 36 & 40 & 31 & 22 & & 0 \\
\hline
\end{tabular}

$B$ brachytherapy, $C N S$ central nervous system, ERT external radiotherapy, $S R S$ stereotactic radiosurgery

versus radiotherapy alone in patients with primary NPC. These trials have been summarized in two meta-analysis studies. Langendijk et al. [10] analyzed 10 randomized clinical studies including 2,450 patients and found a survival benefit of $20 \%$ after 5 years, with similar results for the incidence of local recurrence and distant metastasis. On the other hand, Chua et al. [40] identified two trials with a total of 784 patients for analysis. The addition of induction chemotherapy to radiotherapy was associated with a decrease in relapse by $14.3 \%$ and cancer-related deaths by $12.9 \%$ at 5 years; however, there was no improvement in overall survival.

While in early stages local control and overall survival rates seem similar with or without chemotherapy, this is not the case for advanced disease. For locally advanced T3-T4 tumors, chemotherapy in conjunction with high cumulative doses of external beam radiation therapy followed by endocavitary brachytherapy boost; IMRT and/or stereotactic radiotherapy are the preferred technique for boosting the primary tumor [41]. Furthermore, in a group of 91 patients, for stage III-IV tumors the 3-year local control was $86 \%$ and the overall survival was $72 \%$ with chemotherapy; they were 68 and $35 \%$ without chemotherapy [42].

It is evident that simultaneous chemoradiation in locally advanced primary NPCs results in a large survival benefit. Nevertheless, the efficacy of chemotherapy for recurrent disease, either as the sole treatment or in combination with radiotherapy is uncertain, and its use has been mainly reported in recurrent NPC as palliative treatment.

Gebbia et al. [43] reported that use of cisplatin-based chemotherapy could reach about $65 \%$ of tumor response in recurrent and/or metastatic NPC but the mean survival is only about 11 months. These results might suggest that the recurrent NPC be highly responsive to chemotherapy but survival with chemotherapy alone is poor. It must be emphasized, however, that in most series there are selection bias in that most patients given chemotherapy had other adverse prognostic factors, and chemotherapy is given either before reirradiation because of a more advanced disease, or after the retreatment with radiotherapy because of poor response, but their findings do not support the routine use of chemotherapy [20]. Although different authors have shown an emerging role for concomitant chemoirradiation as the primary treatment of local and regionally advanced NPC, it is uncertain whether it is applicable to retreatment of purely local recurrences, because the high rates of toxicity and potential complications.

\section{Nasopharyngectomy}

Results of surgical treatment and prognostic factors

The role of surgery in the management of malignant tumors involving the nasopharynx and the infratemporal fossa is supported by recent reports. The results of removal of recurrent NPC after radiotherapy failure are encouraging for T1-T3 tumors, while those patients with $\mathrm{T} 4$ stage generally recur locally or die due to development of metastatic disease. Nevertheless, 5 patients out of 14 with stage T4 in the Suárez et al. [44] series were alive, with a follow-up between 1 and 7 years, but none of them had intracranial invasion. At variance, all 5 patients with intracranial involvement died. In other words, the impact on survival of involvement of different areas in stage T4 is not the same, the intracranial extension being the worse prognostic factor. Although some authors have reported encouraging results in cases of intracavernous involvement [45], surgical salvage of recurrences of NPCs with significant intracranial extension usually is not justified.

Analysis of the literature on nasopharyngectomy depicts a $30-55 \%$ overall 5 -year survival, with the largest series consisting of 60 patients [14, 46-56]. The results of some of these series are shown in Table $2[14,47,49,53,56]$.

Teo et al. [14] reported on the treatment results of 20 patients with locally recurrent NPC (mostly rT1 and rT2) 
Table 2 Results with surgery

\begin{tabular}{llllll}
\hline Author & $\begin{array}{l}\text { No. of } \\
\text { cases }\end{array}$ & $\begin{array}{l}\text { Local } \\
\text { control (\%) }\end{array}$ & $\begin{array}{l}\text { 5-year } \\
\text { survival (\%) }\end{array}$ & $\begin{array}{l}\text { Total } \\
\text { complications (\%) }\end{array}$ & $\begin{array}{l}\text { Mortality } \\
(\%)\end{array}$ \\
\hline King et al. [56] & 31 & 53 & 47 & & 0 \\
Wei [47] & 60 & 62 & 49 & 54 & 0 \\
Fee et al. [49] & 37 & 67 & 52 & & 3 \\
Hsu et al. [53] & 60 & 40 & 30 & & 0 \\
Teo et al. [14] & 20 & 40 & 34 & 0 \\
\hline
\end{tabular}

treated with nasopharyngectomy with/without postoperative radiotherapy. This group was compared with other rT1-T2 patients treated mainly with external radiotherapy of $60 \mathrm{~Gy}$ or greater. Far better local control and survival was shown for those receiving nasopharyngectomy than reirradiation. However, $70 \%$ of the patients that underwent surgery also had postoperative radiotherapy due to questionable and/or unclear resection margins. For the subgroup that could be treated adequately with nasopharyngectomy alone, there is a theoretical advantage, as the risk of postradiotherapy complications could be minimized.

In contrast, no significant differences in overall survival were observed between a group of 159 patients who received reirradiation and another group of 22 patients who underwent nasopharyngectomy with or without postoperative radiotherapy. Salvage surgery was associated with improved overall survival only in the subgroup with $\mathrm{T} 1$ to $\mathrm{T} 2$ local failure, but not in the subgroups with $\mathrm{T} 3$ or T4 disease [57].

Wei et al. [47] reported a 5-year actuarial tumor control rate of $62 \%$, and a 5-year actuarial disease-free survival rate of $49 \%$ in a series of 60 patients who had a curative resection.

The importance of postoperative radiotherapy after nasopharyngectomy is stressed by King et al. [56], who found a mean overall survival of 18 months for patients without postoperative radiotherapy, whereas for patients given postoperative radiotherapy the mean overall survival was 60 months. However, postoperative irradiation failed to affect the prognosis significantly in Hsu et al.'s series [53].

When total resection is achieved local recurrence is not very common, but in addition postoperative reirradiation is recommended in patients with positive surgical margins or advanced stage [ $46,47,52]$. If postoperative radiotherapy is required for positive or uncertain margins, the toxicity may be compounded and aggravated. Extensive intradural invasion, cavernous sinus involvement and pharyngobasilar fasciae invasion are considered contraindications for surgery in recurrent NPC [50].

Nasopharyngectomy can be carried out even after a second recurrence but given the small number of patients treated, it is difficult to make definitive conclusions on its effectiveness in reirradiated patients. However, it has been suggested that in appropriately selected patients, the second course of radiation therapy does not contraindicate surgical resection, and, in fact, excellent long-term outcome has been reported. Nevertheless, it increases the morbidity of the procedure, particularly skull base osteomyelitis, and great emphasis should be placed on the reconstruction of the surgical bed, including the use of free flaps [58].

Analysis of clinical prognostic factors in a series of 53 nasopharyngectomies revealed that margin status, adjuvant treatment type and parapharyngeal space involvement were significant impact factors of local control, whereas dura or brain involvement, local recurrence and adjuvant treatment type were significant impact factors of survival [59]. According to To et al. [55] all patients who developed repeat recurrence or died had a high recurrent T-stage tumor, skull base involvement, multiple recurrences, positive surgical margins, or concurrent neck node metastasis.

Indications of surgical approaches

A number of anterior and lateral approaches to neoplasms within the nasopharynx and the infratemporal fossa has been described [46, 48, 52, 53, 60-62]. It is necessary to remove all the related structures in the nasopharynx through a wide approach such as that used in some anterolateral approaches, due to the fact that serial whole-organ section of resected nasopharyngeal specimens showed that $90 \%$ of the tumors affected the eustachian tube cartilage, and over $90 \%$ of the tumors showed extensive submucosal infiltration [63].

For tumors arising from the nasopharynx and extending to neighboring regions, such as nasal cavity, maxillary sinus, sphenoid sinus, and infratemporal fossa, the facial translocation approach can be the simplest and most direct way to expose these areas and to facilitate extensive tumor removal. If the tumor is confined to the nasopharynx or has paranasal, retromaxillary or moderate infratemporal extension, a temporary removal of the anterior, superior and medial walls of the maxillary sinus will suffice (Fig. 1). The procedure is carried out with midfacial degloving in order to avoid facial scars, which in addition allows an excellent exposure [44, 58, 61, 64, 65]. In extensive tumors of the nasopharynx and the infratemporal fossa, especially when 
Fig. 1 a Tumor in the roof of the nasopharynx. b Facial degloving and osteotomies including the anterior, medial and lateral walls of the maxillary sinus. c Once removed the posterior wall access is gained to the nasopharynx and infratemporal fossa. d Reposition of removed bones at the end of procedure
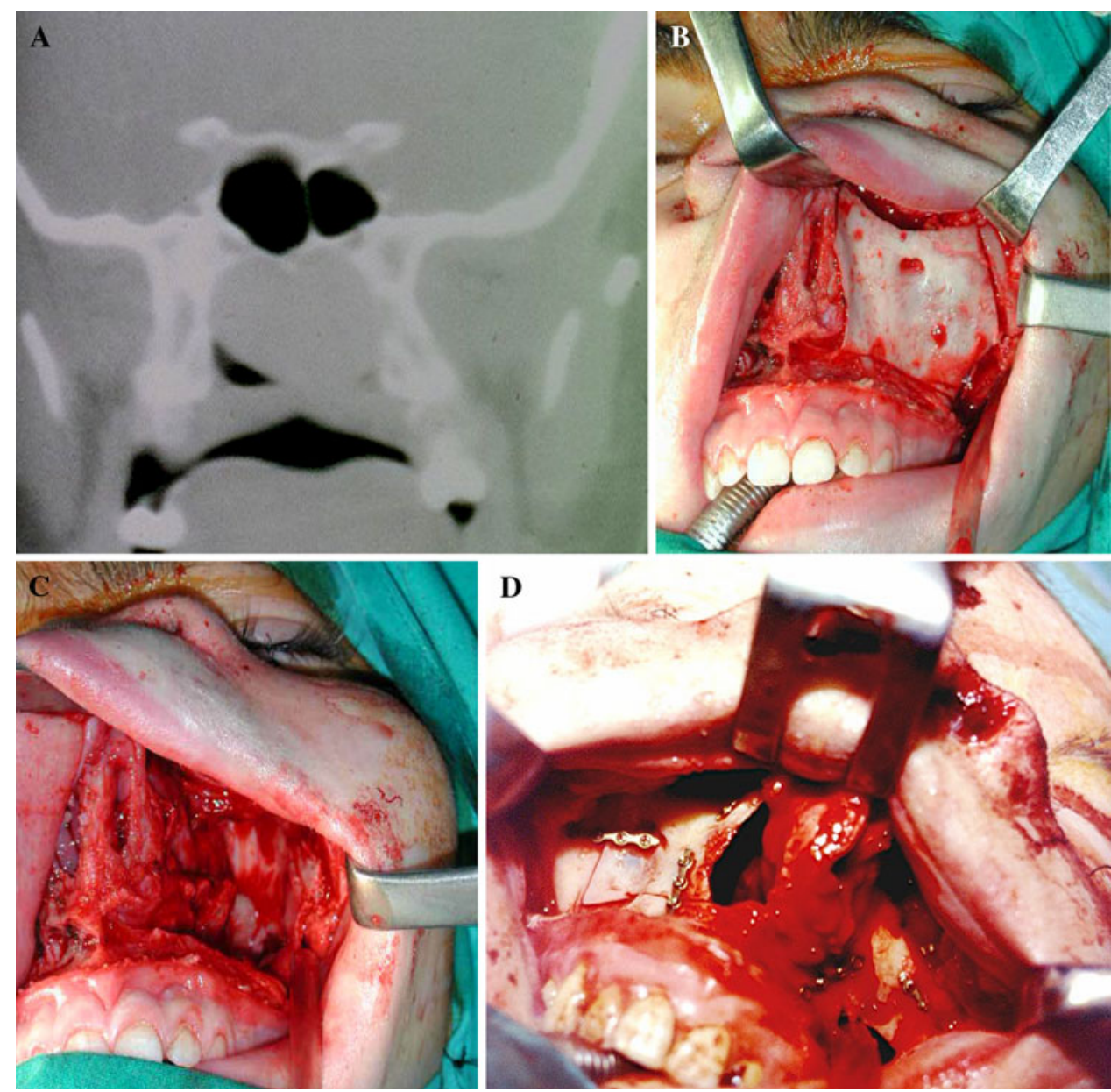

the tumor involves the nasal cavity and the posterior wall of the maxillary sinus, a standard facial translocation approach is the method of choice, allowing the surgeon a better exposure than that obtained by other infratemporal fossa procedures, such the subtemporal-preauricular approach (Figs. 2, 3). In this case the procedure is carried out with midfacial degloving and a hemicoronal-preauricular incision. When a facial translocation is performed, the possibility of osteomyelitis or late bone resorption exists [64]. Open standard facial translocation carries very noticeable sequelae, such as lower lid ectropion, epiphora, medial canthus misalignment, nasolacrimal duct obstruction, frontal muscle palsy, and visible facial scars. Most of these sequelae, with the exception of nasolacrimal obstruction, can be avoided with the use of midfacial degloving combined with a hemicoronal preauricular incision that is hidden in the hair.

The subtemporal-preauricular approach provides a direct access to the nasopharynx and related areas, including the petrous bone, clivus, infratemporal fossa, retropharyngeal area, apex of the orbit, and the sphenoid and maxillary sinuses [62, 66] (Figs. 4, 5). This approach is very versatile, and it may be easily combined with other skull base procedures in selected cases. When necessary, additional drilling of the lateral wall of the orbit, the adjacent greater wing of the sphenoid on the anterior pole of the temporal lobe, and the roof of the infratemporal fossa, permits the exposure of extradural tumors involving the floor of the middle fossa [66].

Transoropalatal, mandibular swing, and maxillary swing approaches are less used now for surgical resection of nasopharyngeal lesions. Generally, the transoropalatal approach is suitable for small tumors centrally located in the midline position of the nasopharynx because the access to the lateral wall of nasopharynx is restricted. This approach causes the least injury and does not affect the face. However, the surgical exposure is usually not satisfactory.

The mandibular swing approach is suitable in patients with severe trismus that makes the transoral approach to access the nasopharynx inadequate or in those patients with oropharyngeal extension (Fig. 6). Moreover, parapharyngeal disease can also be dealt with by this approach. On the other hand, the surgical field is narrow, and secondary injury is often severe.

Wei et al. [46] adopted an anterolateral approach to the nasopharynx with the maxilla swung laterally to provide 

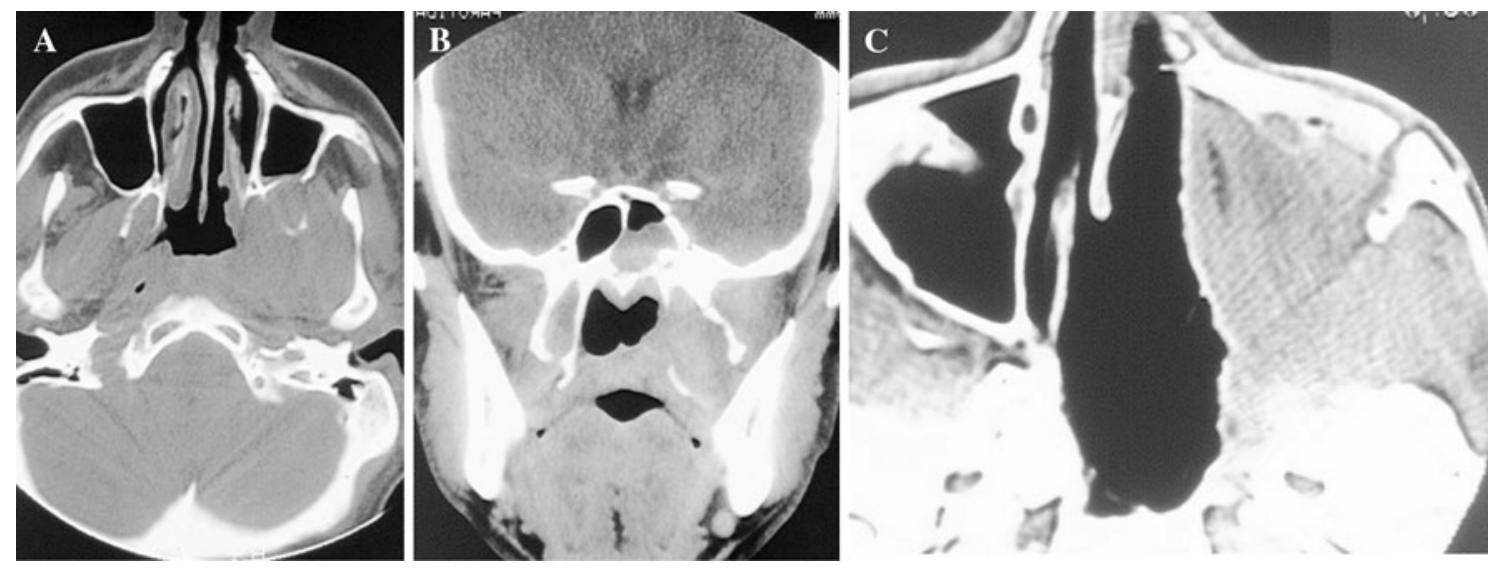

Fig. 2 a Axial CT showing an advanced tumor involving the lateral wall od nasopharynx, infratemporal fossa, pterygoid plates and posterior wall of maxillary sinus. b The coronal CT shows an extension into the sphenoid sinus. c Postoperative CT after a facial translocation approach. The anterior maxillary sinus wall and orbito-zygomatic bones are repositioned and a temporalis muscle flap fills the operative cavity
Fig. 3 Facial translocation approach performed through a facial degloving and a hemicoronal incision. a Translocated facial bones in a single piece. b Bilateral facial degloving. c Reposition of the anterior segment of translocated bones. $\mathbf{d}$ Reposition of the orbito-zygomatic bones and part of the temporalis muscle
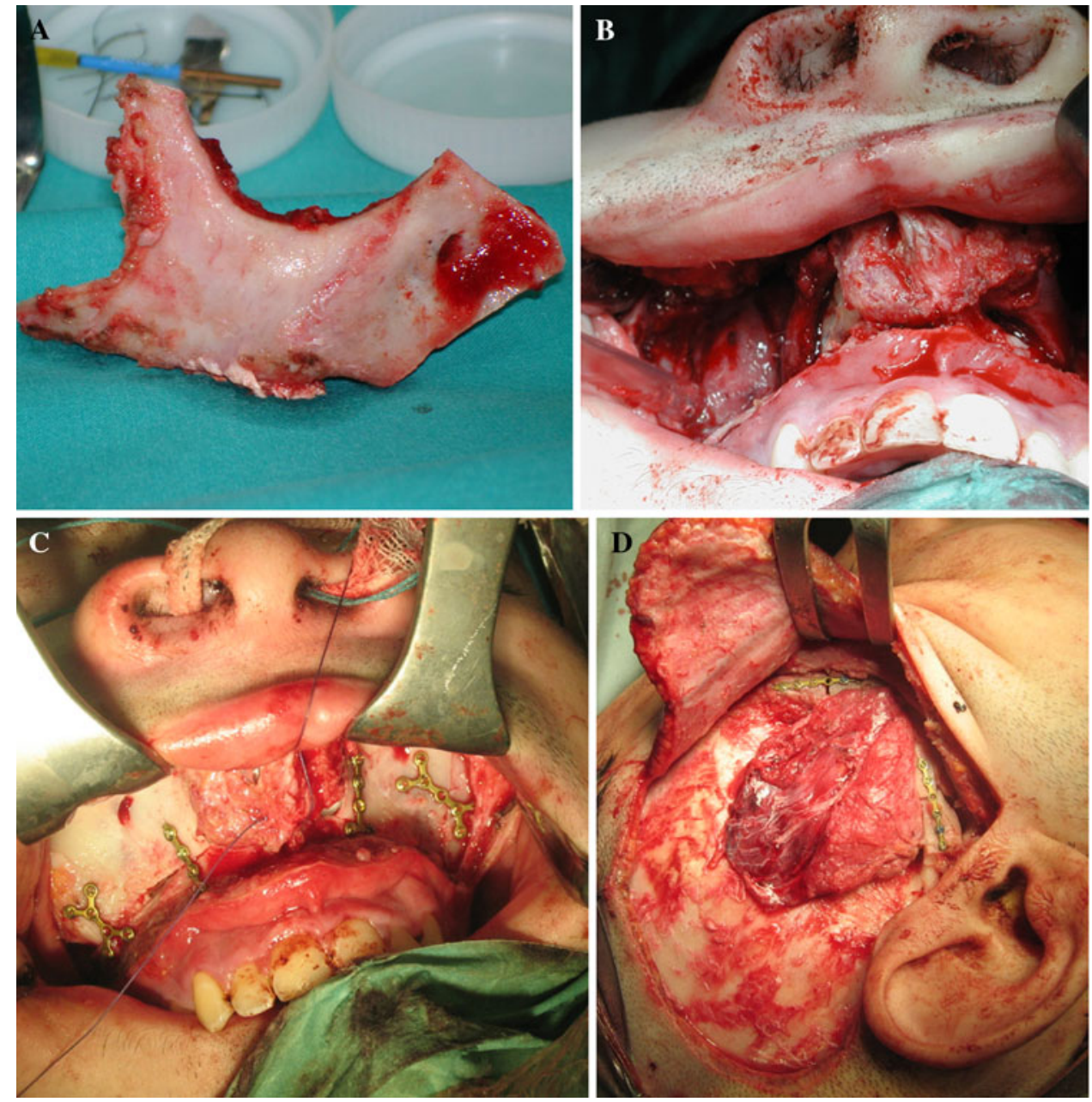

adequate exposure of the nasopharynx, having the drawback of producing noticeable facial incisions. The posterior nasal septum can also be removed for better exposure of the nasopharynx and the paranasopharyngeal space without the necessity of removing the orbital floor. However, the expo- sure of the nasopharynx is somewhat limited to one side of the nasopharynx. In addition, the maxillary bone needs to be freed, and the hard and soft palate have to be split. This approach can potentially result in destructive complications such as maxillary necrosis, palate fistula, and trismus. 

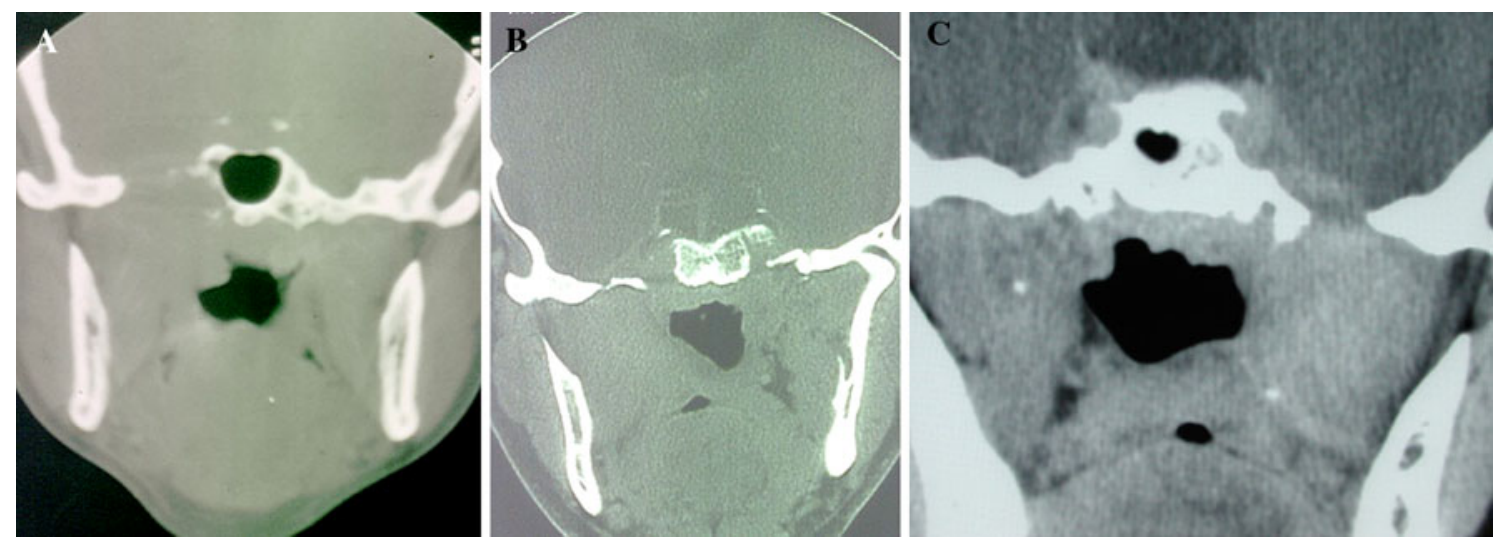

Fig. 4 Different nasopharyngeal recurrent tumors operated by a subtemporal-preauricular approach. a Right lateral wall tumor involving the infratemporal fossa and skull base extradurally. b Right lateral

Fig. 5 Subtemporal-preauricular approach. a Hemicoronalpreauricular incision with optional neck extension.

b Exposition of the orbito-zygomatic complex before its removal. c The nasopharyngectomy is completed. d Reposition of the orbito-zygomatic bones and part of the temporalis muscle wall tumor involving the infratemporal fossa. $\mathbf{c}$ Left lateral wall tumor involving the infratemporal fossa
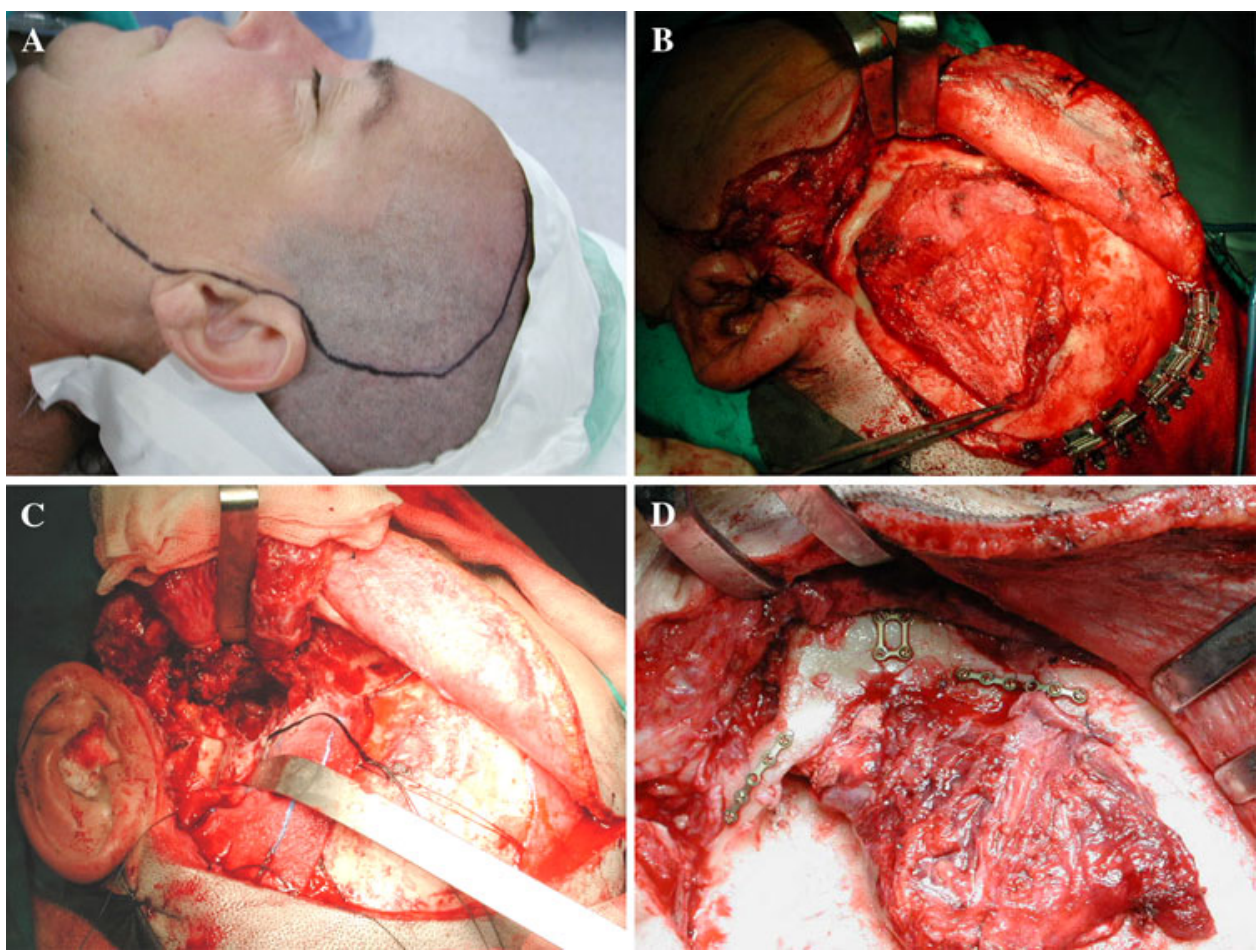

The unsatisfactory surgical exposure and the relatively high frequency of surgical complications using open techniques led to the development of minimally invasive surgical methods that overcomes the limitations of conventional surgery. Currently, use of endoscopic nasopharyngectomy for tumor resection has been reported for a limited number of cases. This novel technique has proven to be minimally invasive and safe for the treatment of recurrent NPC with encouraging short-term outcomes, but further long-term follow-up of the patients is required. Thus, Chen et al. [67] carried out an endoscopic nasopharyngectomy in 37 recurrent NPC, almost all of them with rT1-T2 tumors. No severe complications or deaths resulting from the operation were observed, with the exception of secretory otitis media, 
Fig. 6 Mandibular swing approach. Nasopharyngeal recurrence (a) extended to the oropharynx (b). c Anterior mandibulotomy and approach to the oropharynx and nasopharynx. d Osteosynthesis of the mandible
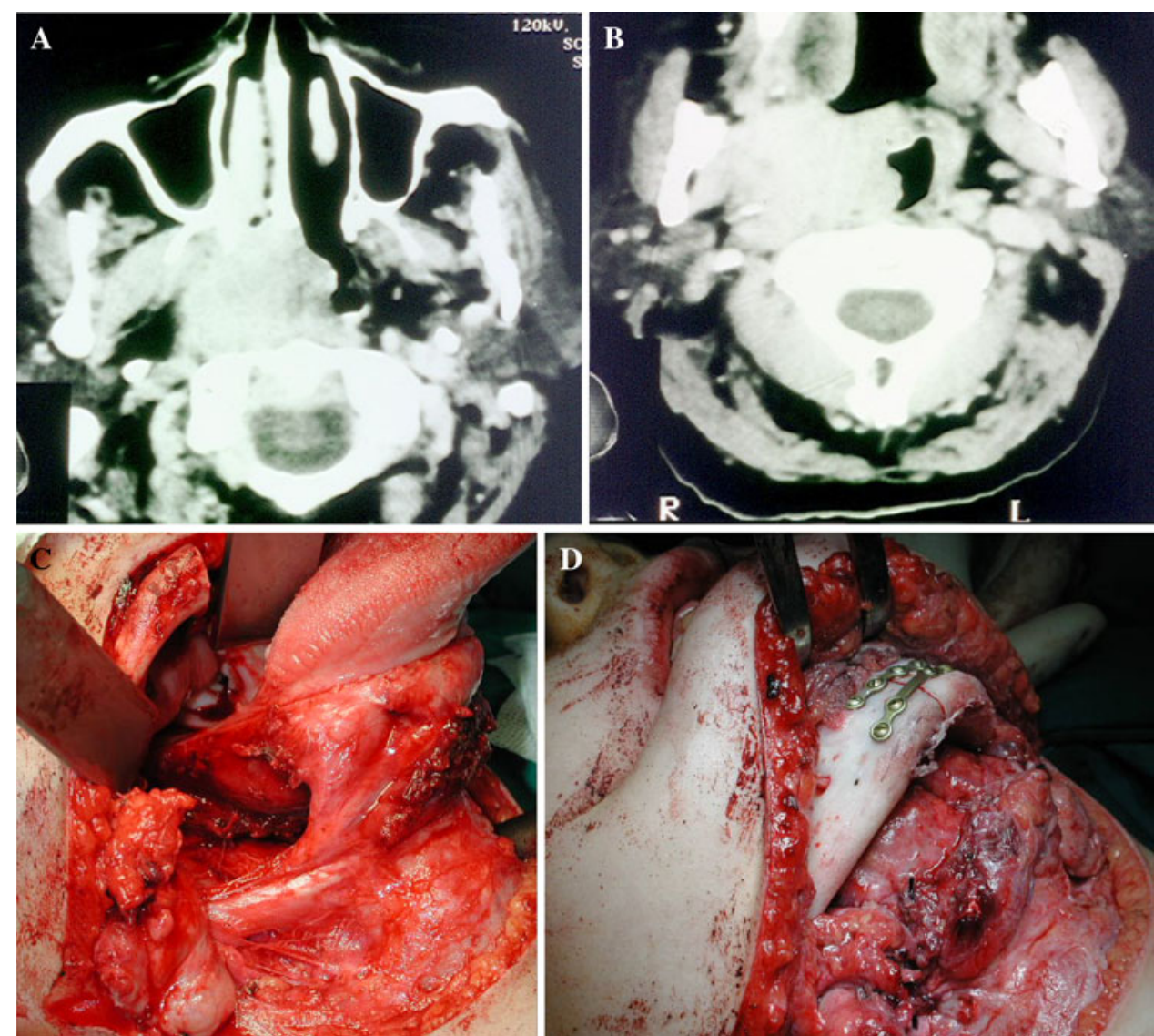

postsurgical complications are less in number and severity than postirradiation ones.

\section{Conclusions}

Retreatment of recurrent NPC using radiotherapy, alone or in combination with other treatment modalities, as well as surgery, can result in long-term loco-regional control and survival in a substantial proportion of patients. Treatment techniques should be highly individualized, and depend on the site and extent of recurrence, previous treatment, and the availability of equipment and expertise.

Better local control and survival rates have been shown for those patients receiving nasopharyngectomy with or without postoperative radiotherapy than reirradiation in a significant part of published series. Nevertheless, for smallvolume recurrent tumors (T1-T2) treated with external radiotherapy, brachytherapy or stereotactic radiosurgery, comparable results to those obtained with surgery have been reported. In contrast, treatment results of advancedstage locally recurrent NPC are generally more satisfactory with surgery than with reirradiation (Fig. 7).

The high incidence of major late complications following reirradiation is of serious concern. Despite new devel-

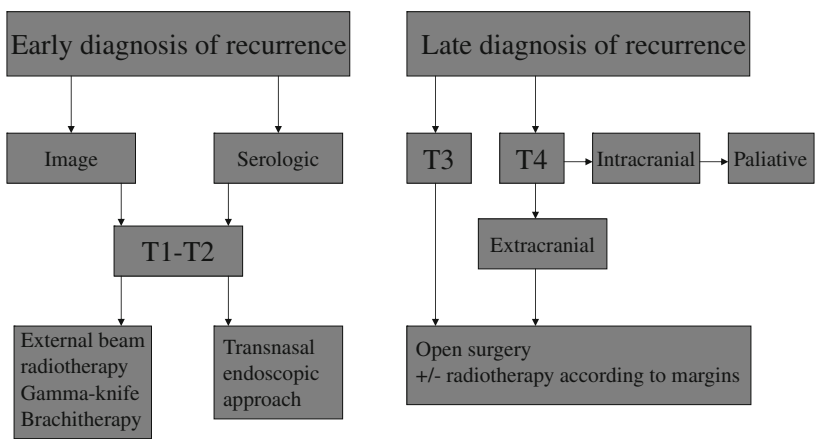

Fig. 7 Guidelines for treatment of recurrent nasopharyngeal cancer

opments in technology there is still a high rate of severe complications following reirradiation, such as brain necrosis, cranial nerve palsies, and catastrophic hemorrhages. On the other hand, the number and severity of complications following surgery, as well as mortality, is smaller. However, questionable or unclear resection margins make necessary the use of postoperative radiotherapy in patients treated surgically; this may increase morbidity and adversely affect the quality of life.

The efficacy of radiosurgery in the reirradiation of locally recurrent NPC remains to be established. Although promising, many published reports on radiosurgery need 
longer follow-up and frequently this technique has been used as a boost for external repeated irradiation. Due to its superior beam characteristics, emerging radiation delivery techniques such as protons have a high potential to increase the therapeutic ratio in locally recurrent NPC with improved local control and/or less toxicity. Endoscopic resection of recurrent NPC offers clear advantages with regard to conventional surgical approaches in terms of morbidity but it lacks long-term follow-up.

Finally, more clinical experiences and trials should be done in China and South Eastern Asian countries randomizing $\mathrm{T} 1+\mathrm{T} 2$ patients to surgery versus radiation therapy comparing outcomes, particularly survival, complications and quality of life, to address fully the role of surgery and the new techniques of reirradiation in salvaging local failure of NPC.

Open Access This article is distributed under the terms of the Creative Commons Attribution Noncommercial License which permits any noncommercial use, distribution, and reproduction in any medium, provided the original author(s) and source are credited.

\section{References}

1. Ragab SM, Erfan FA, Khalifa MA, Korayem EM, Tawfik HA (2008) Detection of local failures after management of nasopharyngeal carcinoma: a prospective, controlled trial. J Laryngol Otol 122:1230-1234

2. Chao SS, Loh KS, Tan LK (2003) Modalities of surveillance in treated nasopharyngeal cancer. Otolaryngol Head Neck Surg 129:61-64

3. Toro C, Rinaldo A, Silver CE, Politi M, Ferlito A (2009) Paraneoplastic syndromes in patients with nasopharyngeal cancer. Auris Nasus Larynx 36:513-520

4. Lee AW, Law SC, Foo W, Poon YF, Cheung FK, Chan DK, Tung SY, Thaw M, Ho JH (1993) Retrospective analysis of patients with nasopharyngeal carcinoma treated during 1976-1985: survival after local recurrence. Int J Radiat Oncol Biol Phys 26:773-782

5. Kwong DL, Wei WI, Cheng AC, Choy DT, Lo AT, Wu PM, Sham JS (2001) Long-term results of radioactive gold grain implantation for the treatment of persistent and recurrent nasopharyngeal carcinoma. Cancer 91:1105-1113

6. Leung TW, Wong VY, Sze WK, Lui CM, Tung SY (2008) Highdose-rate intracavitary brachytherapy boost for early $\mathrm{T}$ stage nasopharyngeal carcinoma. Int J Radiat Oncol Biol Phys 70:361-367

7. Kim K, Wu HG, Kim HJ, Sung MW, Kim KH, Lee SH, Heo DS, Kim HJ, Park CI (2009) Intensity-modulated radiation therapy with simultaneous integrated boost technique following neoadjuvant chemotherapy for locoregionally advanced nasopharyngeal carcinoma. Head Neck 31:1121-1128

8. Palazzi M, Orlandi E, Bossi P, Pignoli E, Potepan P, Guzzo M, Franceschini M, Scaramellini G, Cantù G, Licitra L, Olmi P, Tomatis $S$ (2009) Further improvement in outcomes of nasopharyngeal carcinoma with optimized radiotherapy and induction plus concomitant chemotherapy: an update of the Milan experience. Int $\mathbf{J}$ Radiat Oncol Biol Phys 74:774-780

9. Taheri-Kadkhoda Z, Pettersson N, Björk-Eriksson T, Johansson KA (2008) Superiority of intensity-modulated radiotherapy over three-dimensional conformal radiotherapy combined with brachytherapy in nasopharyngeal carcinoma: a planning study. Br J Radiol 81:397-405
10. Langendijk JA, Leemans CR, Buter J, Berkhof J, Slotman BJ (2004) The additional value of chemotherapy to radiotherapy in locally advanced nasopharyngeal carcinoma: a metaanalysis of the published literature. J Clin Oncol 22:4604-4612

11. Wang CC (1987) Re-irradiation of recurrent nasopharyngeal carcinoma. Treatment techniques and results. Int J Radiat Oncol Biol Phys 13:953-956

12. Pryzant RM, Wendt CD, Delclos L, Peters LJ (1992) Re-treatment of nasopharyngeal carcinoma in 53 patients. Int $\mathrm{J}$ Radiat Oncol Biol Phys 22:941-947

13. Yan JH, Hu YH, Gu XZ (1983) Radiation therapy of recurrent nasopharyngeal carcinoma: report on 219 patients. Acta Radiol Oncol 22:23-28

14. Teo PM, Kwan WH, Chan AT, Lee WY, King WW, Mok CO (1998) How successful is high-dose ( $>$ or $=60 \mathrm{~Gy}$ ) reirradiation using mainly external beams in salvaging local failures of nasopharyngeal carcinoma? Int J Radiat Oncol Biol Phys 40:897-913

15. Chan SC, Ng SH, Chang JT, Lin CY, Chen YC, Chang YC, Hsu CL, Wang HM, Liao CT, Yen TC (2006) Advantages and pitfalls of 18F-fluoro-2-deoxy-D-glucose positron emission tomography in detecting locally residual or recurrent nasopharyngeal carcinoma: comparison with magnetic resonance imaging. Eur J Nucl Med Mol Imaging 33:1032-1040

16. Tsai MH, Shiau YC, Kao CH, Shen YY, Lin CC, Lee CC (2002) Detection of recurrent nasopharyngeal carcinomas with positron emission tomography using 18-fluoro-2-deoxyglucose in patients with indeterminate magnetic resonance imaging findings alter radiotherapy. J Cancer Res Clin Oncol 128:279-282

17. Yen RF, Hung RL, Pan MH, Wang YH, Huang KM, Lui LT, Kao $\mathrm{CH}$ (2003) 18-fluoro-2-deoxyglucose positron emission tomography in detecting residual/recurrent nasopharyngeal carcinomas and comparison with magnetic resonance imaging. Cancer 98:283-287

18. Ng SH, Joseph CT, Chan SC, Ko SF, Wang HM, Liao CT, Chang YC, Lin WJ, Fu YK, Yen TC (2004) Clinical usefulness of 18FFDG PET in nasopharyngeal carcinoma patients with questionable MRI findings for recurrence. J Nucl Med 45:1669-1676

19. Comoretto M, Balestreri L, Borsatti E, Cimitan M, Franchin G, Lise M (2008) Detection and restaging of residual and/or recurrent nasopharyngeal carcinoma after chemotherapy and radiation therapy: comparison of MR imaging and FDG PET/CT. Radiology 249:203-211

20. Chang JT, See LC, Liao CT, Ng SH, Wang CH, Chen IH, Tsang NM, Tseng CK, Tang SG, Hong JH (2000) Locally recurrent nasopharyngeal carcinoma. Radiother Oncol 54:135-142

21. Cabanillas R, Llorente JL, Estelrrich PM, Martínez-Cassati ME, Franco V, Suárez C (2005) Functional and oncologic results of the surgical salvage in the management of recurrent nasopharyngeal carcinomas. Acta Otorrinolaringol Esp 56:416-422 [in Spanish]

22. Hwang JM, Fu KK, Phillips TL (1998) Results and prognostic factors in the retreatment of locally recurrent nasopharyngeal carcinoma. Int J Radiat Oncol Biol Phys 41:1099-1111

23. Leung TW, Tung SY, Sze WK, Sze WM, Wong VY, Wong CS, O SK (2000) Salvage radiation therapy for locally recurrent nasopharyngeal carcinoma. Int J Radiat Oncol Biol Phys 48:1331-1338

24. Lee AW, Foo W, Law SC, Poon YF, Sze WM, O SK, Tung SY, Lau WH (1997) Reirradiation for recurrent nasopharyngeal carcinoma: factors affecting the therapeutic ratio and ways for improvement. Int J Radiat Oncol Biol Phys 38:43-52

25. Lee N, Xia P, Quivey JM, Sultanem K, Poon I, Akazawa C, Akazawa P, Weinberg V, Fu KK (2002) Intensity-modulated radiotherapy in the treatment of nasopharyngeal carcinoma: an update of the UCSF experience. Int J Radiat Oncol Biol Phys 53:12-22

26. Kam MK, Teo PM, Chau RM, Cheung KY, Choi PH, Kwan WH, Leung SF, Zee B, Chan AT (2004) Treatment of nasopharyngeal carcinoma with intensity-modulated radiotherapy: the Hong Kong experience. Int J Radiat Oncol Biol Phys 60:1440-1450 
27. Lu TX, Mai WY, The BS, Zhao C, Han F, Huang Y, Deng XW, Lu LX, Huang SM, Zeng ZF, Lin CG, Lu HH, Chiu JK, Carpenter LS, Grant WH 3rd, Woo SY, Cui NJ, Butler EB (2004) Initial experience using intensity-modulated radiotherapy for recurrent nasopharyngeal carcinoma. Int J Radiat Oncol Biol Phys 58:682687

28. Chua DT, Sham JS, Leung LH, Au GK (2005) Re-irradiation of nasopharyngeal carcinoma with intensity-modulated radiotherapy. Radiother Oncol 77:290-294

29. Choy D, Sham JST, Wei WI, Ho CM, Wu PM (1993) Transpalatal insertion of radioactive gold grain for the treatment of persistent and recurrent nasopharyngeal carcinoma. Int J Radiat Oncol Biol Phys 25:505-512

30. Zheng XK, Chen LH, Chen YQ, Deng XG (2004) Three-dimensional conformal radiotherapy versus intracavitary brachytherapy for salvage treatment of locally persistent nasopharyngeal carcinoma. Int J Radiat Oncol Biol Phys 60:165-170

31. Chua DT, Sham JS, Hung KN, Leung LH, Au GK (2006) Predictive factors of tumor control and survival after radiosurgery for local failures of nasopharyngeal carcinoma. Int J Radiat Oncol Biol Phys 66:1415-1421

32. Low JS, Chua ET, Gao F, Wee JT (2006) Stereotactic radiosurgery plus intracavitary irradiation in the salvage of nasopharyngeal carcinoma. Head Neck 28:321-329

33. Chua DT, Wei WI, Sham JS, Hung KN, Au GK (2007) Stereotactic radiosurgery versus gold grain implantation in salvaging local failures of nasopharyngeal carcinoma. Int J Radiat Oncol Biol Phys 69:469-474

34. Pai PC, Chuang CC, Wei KC, Tsang NM, Tseng CK, Chang CN (2002) Stereotactic radiosurgery for locally recurrent nasopharyngeal carcinoma. Head Neck 24:748-753

35. Kocher M, Voges J, Staar S, Treuer H, Sturm V, Mueller RP (1998) Linear accelerator radiosurgery for recurrent malignant tumors of the skull base. Am J Clin Oncol 21:18-22

36. Xiao J, Xu G, Miao Y (2001) Fractionated stereotactic radiosurgery for 50 patients with recurrent or residual nasopharyngeal carcinoma. Int J Radiat Oncol Biol Phys 51:164-170

37. Wu SX, Chua DT, Deng ML, Zhao C, Li FY, Sham JS, Wang HY, Bao Y, Gao YH, Zeng ZF (2007) Outcome of fractionated stereotactic radiotherapy for 90 patients with locally persistent and recurrent nasopharyngeal carcinoma. Int J Radiat Oncol Biol Phys 69:761-769

38. Chua DT, Wu SX, Lee V, Tsang J (2009) Comparison of single versus fractionated dose of stereotactic radiotherapy for salvaging local failures of nasopharyngeal carcinoma: a matched-cohort analysis. Head Neck Oncol 1:13

39. Widesott L, Pierelli A, Fiorino C, Dell'Oca I, Broggi S, Cattaneo GM, Di Muzio N, Fazio F, Calandrino R, Schwarz M (2008) Intensity-modulated proton therapy versus helical tomotherapy in nasopharynx cancer: planning comparison and NTCP evaluation. Int J Radiat Oncol Biol Phys 72:589-596

40. Chua DT, Ma J, Sham JS, Mai HQ, Choy DT, Hong MH, Lu TX, Min HQ (2005) Long-term survival after cisplatin-based induction chemotherapy and radiotherapy for nasopharyngeal carcinoma: a pooled data analysis of two phase III trials. J Clin Oncol 23:11181124

41. Levendag PC, Lagerwaard FJ, de Pan C, Noever I, van Nimwegen A, Wijers O, Nowak PJ (2002) High-dose, high-precision treatment options for boosting cancer of the nasopharynx. Radiother Oncol 63:67-74

42. Levendag PC, Lagerwaard FJ, Noever I, dePan C, van Nimwegen A, Wijers O, Schmitz PI, van Dieren E, Nowak PJ (2002) Role of endocavitary brachytherapy with or without chemotherapy in cancer of the nasopharynx. Int J Radiat Oncol Biol Phys 52:755-768

43. Gebbia V, Zerillo G, Restivo G, Speciale R, Cupido G, Lo Bue P, Ingria F, Gallina S, Spatafora G, Testa A, Cannata G, Cimino A,
Gebbial N (1993) Chemotherapeutic treatment of recurrent and/or metastatic nasopharyngeal carcinoma: a retrospective analysis of 40 cases. Br J Cancer 68:191-194

44. Suárez C, Llorente JL, Muñoz C, García LA, Rodrigo JP (2004) Facial translocation approach in the management of nasopharyngeal and sinonasal tumors. Laryngoscope 114:1047-1051

45. Donald PJ, Boggan J (1990) Sphenoidal and cavernous sinus resection for tumor. J Otolaryngol 19:122-129

46. Wei WI, Ho CM, Yuen PW, Fung CF, Sham JS, Lam KH (1995) Maxillary swing approach for resection of tumors in and around the nasopharynx. Arch Otolaryngol Head Neck Surg 121:638-642

47. Wei WI (2000) Salvage surgery for recurrent primary nasopharyngeal carcinoma. Crit Rev Oncol Hematol 33:91-98

48. Fee WE, Robertson JB, Goffinet DR (1991) Long-term survival after surgical resection for recurrent nasopharyngeal cancer after radiotherapy failure. Arch Otolaryngol Head Neck Surg 117:1233-1236

49. Fee W Jr, Moir M, Choi EC, Goffinet D (2002) Nasopharyngectomy for recurrent nasopharyngeal cancer: a 2- to 17-year follow-up. Arch Otolaryngol Head Neck Surg 128:280-284

50. Hao SP, Tsang NM, Chang CN (2002) Salvage surgery for recurrent nasopharyngeal carcinoma. Arch Otolaryngol Head Neck Surg 128:63-67

51. Hao SP, Pan WL, Chang CN, Hsu YS (2003) The use of the facial translocation technique in the management of tumors of the paranasal sinuses and skull base. Otolaryngol Head Neck Surg 128:571-575

52. Shu CH, Cheng H, Lirng JF, Chang FC, Chao Y, Chi KH, Yen SH (2000) Salvage surgery for recurrent nasopharyngeal carcinoma. Laryngoscope 110:1483-1488

53. Hsu MM, Ko JY, Sheen TS, Chang YL (1997) Salvage surgery for recurrent nasopharyngeal carcinoma. Arch Otolaryngol Head Neck Surg 123:305-309

54. Tu GY, Hu YH, Xu GZ, Ye M (1988) Salvage surgery for nasopharyngeal carcinoma. Arch Otolaryngol Head Neck Surg 114:328-329

55. To EW, Lai EC, Cheng JH, Pang PC, Williams MD, Teo PM (2002) Nasopharyngectomy for recurrent nasopharyngeal carcinoma: a review of 31 patients and prognostic factors. Laryngoscope 112:1877-1882

56. King WW, Ku PK, Mok CO, Teo PM (2000) Nasopharyngectomy in the treatment of recurrent nasopharyngeal carcinoma: a twelveyear experience. Head Neck 22:215-222

57. Yu KH, Leung SF, Tung SY, Zee B, Chua DT, Sze WM, Law SC, Kam MK, Leung TW, Sham JS, Lee AW, Au JS, Hui EP, Sze WK, Cheng AC, Yau TK, Ngan RK, Wong FC, Au GK, Chan AT (2005) Survival outcome of patients with nasopharyngeal carcinoma with first local failure: a study by the Hong Kong Nasopharyngeal Carcinoma Study Group. Head Neck 27:397-405

58. Ibrahim HZ, Moir MS, Fee WW (2002) Nasopharyngectomy after failure of 2 courses of radiation therapy. Arch Otolaryngol Head Neck Surg 128:1196-1197

59. Hao SP, Tsang NM, Chang KP, Hsu YS, Chen CK, Fang KH (2008) Nasopharyngectomy for recurrent nasopharyngeal carcinoma: a review of 53 patients and prognostic factors. Acta Otolaryngol 128:473-481

60. Krespi YP (1989) Lateral skull base surgery for cancer. Laryngoscope 99:514-524

61. Janecka IP (1995) Classification of facial translocation approach to the skull base. Otolaryngol Head Neck Surg 112:579-585

62. Sekhar LN, Schramm VL, Jones NF (1987) Subtemporal-preauricular infratemporal fossa approach to large lateral and posterior cranial base neoplasms. J Neurosurg 67:488-499

63. Wei WI (2001) Nasopharyngeal cancer: current status of management. Arch Otolaryngol Head Neck Surg 127:766-769 
64. Hao SP (2001) Facial translocation approach to the skull base: the viability of translocated facial bone graft. Otolaryngol Head Neck Surg 124:292-296

65. Chang KP, Hao SP, Tsang NM, Ueng SH (2004) Salvage surgery for locally recurrent nasopharyngeal carcinoma-a 10-year experience. Otolaryngol Head Neck Surg 131:497-502

66. Llorente JL, Nazar G, Cabanillas R, Fernández de León R, Suárez C (2006) Subtemporal-preauricular approach in the management of infratemporal and nasopharyngeal tumors. J Otolaryngol 35:173-179

67. Chen MY, Wen WP, Guo X, Yang AK, Qian CN, Hua YJ, Wan XB, Guo ZM, Li TY, Hong MH (2009) Endoscopic nasopharyngectomy for locally recurrent nasopharyngeal carcinoma. Laryngoscope 119:516-522 